\title{
Lessons learned from a phase II clinical trial of laparoscopic HIPEC for gastric cancer
}

\author{
Brian Badgwell ${ }^{1,6} \cdot$ Mariela Blum $^{2} \cdot$ Prajnan Das $^{3} \cdot$ Jeannelyn Estrella $^{4}$. \\ Xuemei Wang ${ }^{5} \cdot$ Keith Fournier $^{1} \cdot$ Richard Royal $^{1} \cdot$ Paul Mansfield $^{1}$. \\ Jaffer Ajani ${ }^{2}$
}

Received: 7 April 2017/Accepted: 7 June 2017/Published online: 22 June 2017

(C) Springer Science+Business Media, LLC 2017

\begin{abstract}
Background Over the last two decades, intraperitoneal chemotherapy has been found to have activity for select subgroups of patients with carcinomatosis from colon, ovarian, appendiceal, and recently, gastric origins. However, there is little data to support an aggressive surgical approach of cytoreduction (debulking) and hyperthermic intraperitoneal perfusion with chemotherapy (HIPEC) for patients with gastric cancer and positive cytology or carcinomatosis. The morbidity and mortality rates of cytoreduction and HIPEC, in combination with gastrectomy, are significant and the survival rates of this approach may not extend beyond that of treatment with systemic
\end{abstract}

Presented at the SAGES 2017 Annual Meeting, March 22-25, 2017, Houston, Texas.

Electronic supplementary material The online version of this article (doi:10.1007/s00464-017-5668-9) contains supplementary material, which is available to authorized users.

Brian Badgwell

bbadgwell@mdanderson.org

1 Departments of Surgical Oncology, The University of Texas MD Anderson Cancer Center, Houston, TX, USA

2 Departments of Gastrointestinal Medical Oncology, The University of Texas MD Anderson Cancer Center, Houston, TX, USA

3 Departments of Radiation Oncology, The University of Texas MD Anderson Cancer Center, Houston, TX, USA

4 Departments of Pathology, The University of Texas MD Anderson Cancer Center, Houston, TX, USA

5 Departments of Biostatistics, The University of Texas MD Anderson Cancer Center, Houston, TX, USA

6 Department of Surgical Oncology, Unit 1484 MD Anderson Cancer Center, 1400 Pressler St., Houston, TX 77030, USA chemotherapy. The objective of this clinical trial, therefore, was to perform HIPEC in a neoadjuvant fashion via a minimally invasive approach without cytoreduction for patients with gastric cancer and positive cytology or low volume carcinomatosis. Patients found to have resolution of all extra-gastric disease are then candidates for gastrectomy.

Methods Patients with gastric and gastroesophageal adenocarcinoma and positive peritoneal cytology or radiologically-occult carcinomatosis that have completed treatment with systemic chemotherapy were offered participation in the study.

Results We have performed 38 laparoscopic HIPEC procedures in 19 patients. Laparoscopic HIPEC consists of Mitomycin C $30 \mathrm{mg}$ and Cisplatin $200 \mathrm{mg}$ in 3-7 L of infusate circulated using an extracorporeal circulation device at a flow rate of $700-1500 \mathrm{~mL} /$ minute for $60 \mathrm{~min}$. The Laparoscopic HIPEC procedure may be performed up to five times. In this video, we sought to present the surgical technique refined during our development and completion of this Phase II clinical trial (NCT02092298).

Conclusion The purpose of this presentation is to (1) demonstrate the technique of laparoscopic HIPEC and (2) review the surgical lessons learned from performing multiple HIPEC procedures prior to attempted gastrectomy.

Keywords Laparoscopic HIPEC · Gastric cancer · Carcinomatosis $\cdot$ Positive cytology $\cdot$ Perfusion

Compliance with ethical standards

Disclosures Drs. Badgwell, Blum, Das, Estrella, Wang, Fournier, Royal, Mansfield, and Ajani have no conflicts of interest or financial ties to disclose. 